удк 62-50

А. А. Воробьев

Санкт-Петербургский институт информатики

и автолатизации Российской академии наук,

г. Санкт-Петербург, Российская Федерация

А. В. Данеев

Иркутский государственный университет путей сообщения, г. Иркутск, Российская Федерация

\title{
ОРГАНИЗАЦИЯ ДЕЯТЕЛЬНОСТИ ПРЕДПРИЯТИЯ ПО УПРАВЛЕНИЮ ЖИЗНЕННЫМ ЦИКЛОМ СЛОЖНЫХ НАУКОЕМКИХ ИЗДЕЛИЙ
}

\begin{abstract}
АНнотАцИЯ. Планирование деятельности предприятий, выпускающих сложную наукоемкую продукцию, является сложной задачей в силу большого числа трудно формализуемых факторов и процессов, обуславливающих необходимость принятия решений в условиях неопределенности. В то же время функционирование значительных по производственным мощностям и людским ресурсам предприятий нередко усложняется свойственной нынешнему времени высокой динамикой изменения или корректировки заказов на выпускаемую продукцию, определяющей возможности «выживания» и развития организаций. В статье рассматривается проблема создания системы управления, причем адаптивного управления (САУ), процессами жизненного цикла (ЖЖЦ) наукоемких изделий, отличающуюся отсутствием ограничений на детерминированность и замкнутость множества входных переменных, что является типичным для задач теории САУ. Решение этой проблемы позволит планировать работу предприятий в долгосрочной перспективе для САУ ЖЖД производимой наукоемкой продукции, а также оптимальное применение производственных и людских ресурсов в среднесрочной и краткосрочной перспективе.

кЛЮчЕВЫЕ слОВА. Планирование деятельности предприятия; наукоемкая продукция; жизненный цикл; управление экономическими процессами.

ИНФОРМАЦИЯ О СТАТЬЕ. Дата поступления 31 октября 2017 г.; дата принятия к печати 19 декабря 2017 г.; дата онлайн-размещения 29 декабря 2017 г.

ФИНАНСИРОВАНИЕ. Работа выполнена в рамках бюджетной темы № 0073-20140009.
\end{abstract}

A. A. Vorobyev

St. Petersburg Institute of Computer Science and Automation of Russian Academy of Sciences, Saint-Petersburg, Russian Federation

A. V. Daneyev

Irkutsk State Railway Engineering University, Irkutsk, Russian Federation

\section{ACTIVITY ARRANGEMENT OF THE ENTERPRISE IN MANAGING THE LIFE CYCLE OF COMPLEX HIGH-TECH PRODUCTS}

\begin{abstract}
Activity planning for enterprises producing complex high-tech products is a difficult problem due to many factors and processes that are difficult to formalize and that cause the necessity of making decisions in the context of uncertainty. At the same time, the functioning of enterprises that are significant in terms of production capacities and human resources is often complicated by high dynamics of changes or adjustments in orders for manufactured products that determine opportunities for "survival" and development of organizations. The article considers the problem of creating an adaptive control system (ACS), life cycle processes (LC) of high-tech products, which is distinguished by absence of restrictions on determinism and circularity
\end{abstract}

\section{Baikal Research Journal}


of input variables, which is typical for the problems of the ACS theory. The solution of this problem will allow to plan the work of enterprises in the long-term prospect for automated control systems of high-tech products, as well as the optimal use of production and human resources in the medium and short-term prospects.

KEYWORDS. Planning of enterprise activity; high technology products; life cycle; management of economic processes.

ARTICLE INFO. Received October 31, 2017; accepted December 19, 2017; available online December 29, 2017.

ACKNOWLEDGEMENTS. The work was performed under the budget theme № 00732014-0009.

Эффективное планирование деятельности предприятия на различные прогнозные периоды является основой его конкурентоспособности и инновационной привлекательности. Для многих предприятий, выпускающих сложную наукоемкую продукцию, планирование деятельности является весьма сложной задачей, прежде всего, в силу большого числа трудно формализуемых факторов и процессов, обуславливающих необходимость принятия решений в условиях неопределенности. Более того, функционирование значительных по производственным мощностям и людским ресурсам предприятий нередко усложняется свойственной нынешнему времени высокой динамикой изменения или корректировки заказов на выпускаемую продукцию, определяющей возможности «выживания» и развития организаций. Указанные обстоятельства затрудняют и долгосрочное планирование работы предприятий, и оптимальное применение производственных возможностей и людских ресурсов в среднесрочной и краткосрочной перспективе. Наиболее остро эта вопрос встает в деятельности современных предприятий военно-промышленного комплекса, нередко сочетающих в едином хозяйствующем субъекте (юридическом лице) возможности проектирования, проведения испытаний, производства и сопровождения эксплуатации изделий.

Традиционно жизненный цикл наукоемкой продукции можно описать применяя разграничение на этапы и стадии, охватывающие процессы создания, производства и эксплуатации изделия, причем могут различаться в зависимости от специфики выпускаемых образцов (рис. 1), [1].

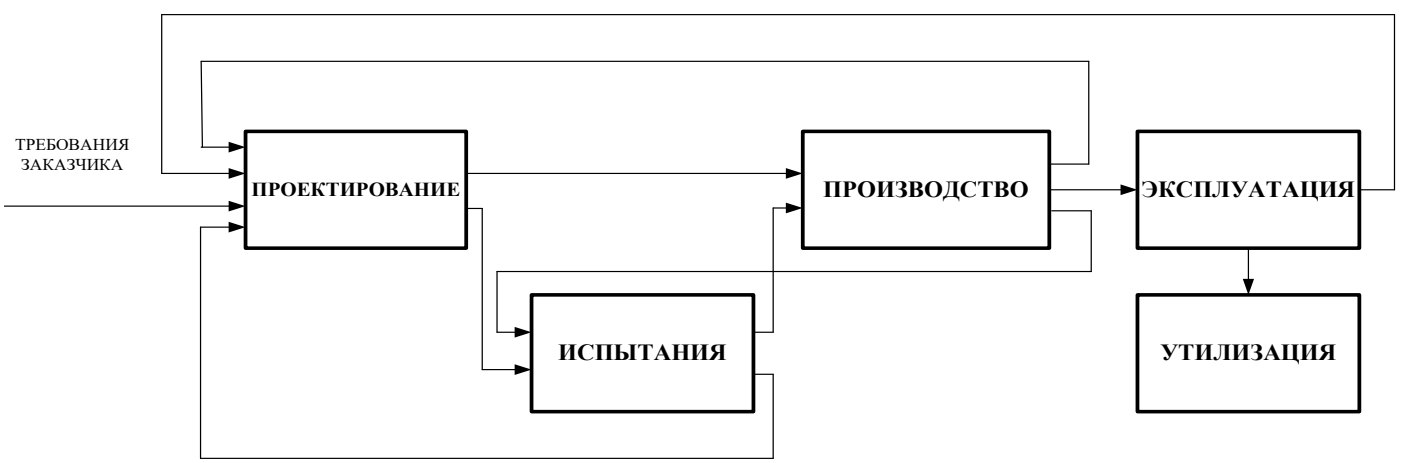

Рис. 1. Стадии (этапы) жизненного ицкла изделий

Как обосновано в [2], исследования в области организации управления жизненным циклом изделий до сих пор ограничиваются, преимущественно, вопросами информационной поддержки начальных стадий жизненного цикла. Основные усилия продолжают направляться на разработку таких технологий, как CRM, CPC, CAD/CAM, CAE, MRP I, MRP-II, PDM, ERP, SCM и др. Поэтому основопо-

\section{Baikal Research Journal}


лагающие научно-методические вопросы организации управления жॄЦ изделий в целом, связанные с постановкой задач для различных стадий управления, информационным обеспечением этого процесса, экономическими аспектами мало исследовались в научной литературе.

В качестве примера в $[2 ; 3]$ приводится следующая динамика изменения определяющих конкурентоспособность предприятия показателей при комплексном управлении совокупностью взаимосвязанных производственных, технологических и экономических процессов:

- сокращение до 40-60 \% работы по технической подготовке и начала выпуска новых изделий;

- уменьшение времени разработки изделий за счет параллельного производства наукоемких изделий несколькими предприятиями;

- сокращение до 25-75 \% сроков ввода изделий в эксплуатацию;

- сокращение эксплуатационных затрат до 40-60\%.

- увеличение до 30 \% мощности по объему выпуска изделий в плановый период;

- повышение качества изделий за счет значительного сокращения числа ошибок и доработок в конструкторско-технологической документации;

- снижение до 2-3 раз количества брака и конструктивно-производственных недостатков.

Вместе с тем, комплексное управление процессами жизненного цикла изделий имеет целью не только общее сокращение издержек на разработку, производство и эксплуатацию выпускаемой продукции, но и предельно возможную унификацию этих процессов, обеспечивающую оперативное достижение заданных требований и характеристик при создании и дальнейшую бесперебойную эксплуатацию изделий. Наиболее ответственными среди всех стадий жизненного цикла сложных наукоемких изделий являются процедуры проектирования (см. рис. 1).

В связи с прогрессивно возрастающей сложностью выпускаемой ролдукции, повышением тактико-технических параметров изделий нужные проектные расчеты оказываются более сложными и трудоемкими, к тому же число требующих проверки вариантов проектирования новых изделий постоянно возрастает. Кроме того, в ряде случаев в ходе проектирования уточняются требования в части эксплуатационно-технических характеристик изделий. Вследствие этого нередко имеют место существенное увеличение сроков и объемов работ в процессе проектирования сложных изделий, а также несоответствие представляемых на испытания опытных образцов изделий заданным требованиям. Перечисленные трудности проектирования сложных изделий определили бурное развитие технологий проектирования с широким применением средств автоматизации рутинных и трудоемких проектных процессов, применением математического моделирования, применением современных методов многовариантных расчетов и векторной оптимизации. Проводимые в этой области исследования позволят в ближайшей перспективе трансформировать существующие технологии проектирования в интересах комплексного управления процессами жизненного цикла парком наукоемких изделий.

Основные функции перспективной единой системы управления жизненным циклом изделий представлены на рис. 2. Участниками процессов управления традиционно являются заказчик, разработчик-изготовитель (рассматриваемый в едином юридическом лице) и эксплуатант. Задачи основных участников процесса управления жизненным циклом парка изделий в общем виде определены в действующих стандартах и нормативных документах и приведены в табл. 1.

\section{Baikal Research Journal}

электронный научный журнал Байкальского государственного университета 


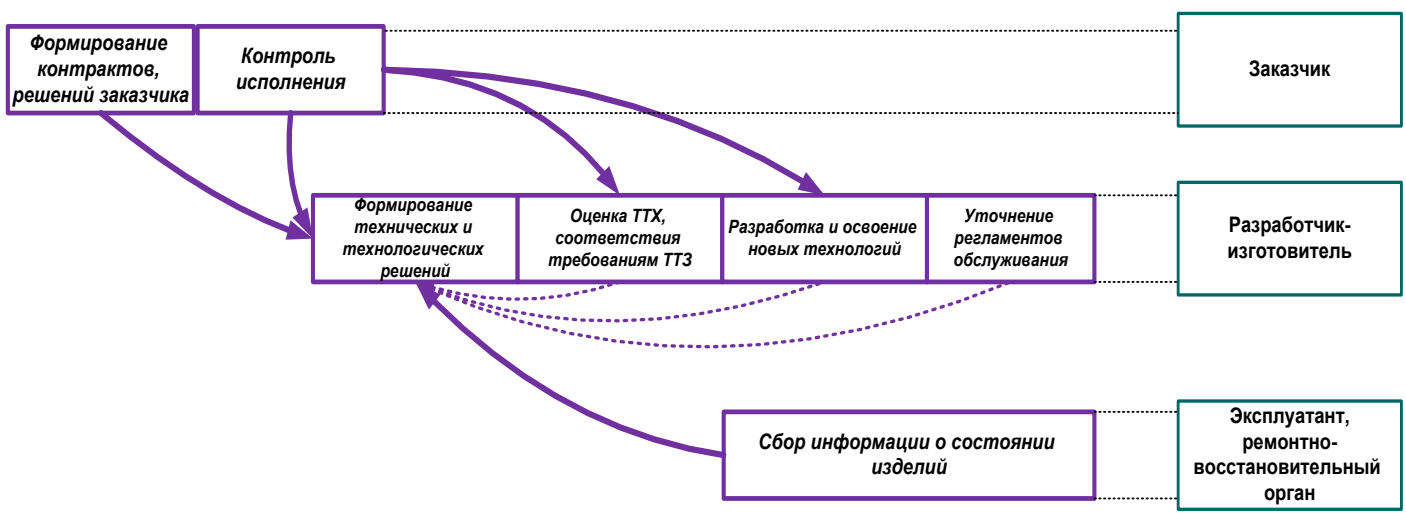

Рисунок 2. Функиии колплексного управления жизненным ииклом изделия

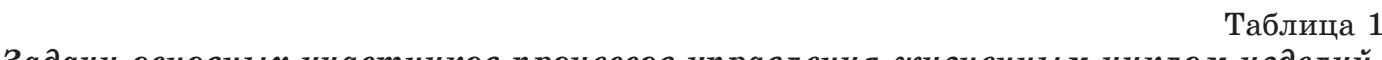
Задачи основных участников процессов управления жизненным циклом изделий

\begin{tabular}{|c|l|l|}
\hline $\begin{array}{c}\text { № } \\
\text { п/п }\end{array}$ & $\begin{array}{l}\text { Наимено- } \\
\text { вание }\end{array}$ & \multicolumn{1}{c|}{ Решаемые задачи } \\
\hline \multirow{2}{*}{2} & Заказчик & $\begin{array}{l}\text { - задание технического облика; } \\
\text { - определение тактико-технических характеристик; } \\
\text { - установление объема парка изделий; } \\
\text { - задание сроков исполнения контрактов }\end{array}$ \\
\hline \multirow{2}{*}{$\begin{array}{l}\text { Разработ- } \\
\text { чик-изг- } \\
\text { товитель }\end{array}$} & $\begin{array}{l}\text { - сазработка и оптимизация конструкции изделий; } \\
\text { - оценка и обеспечение соответствия изделия заданным тактико-техниче- } \\
\text { ским характеристикам; } \\
\text { - снижение производстенных издержек; } \\
\text { - каталогизация изделия; } \\
\text { - разработка рабочей и конструкторской документации; } \\
\text { - контроль изделий в эксплуатации (через гарантийный и авторский надзор); } \\
\text { - совершенствование эксплуатационных мероприятий }\end{array}$ \\
\hline Эксплуа- & $\begin{array}{l}\text { - техническое обслуживание; } \\
\text { - проведение регламентных работ; } \\
\text { - текущий ремонт; } \\
\text { - сбор и передача разработчику сведений о техническом состоянии } \\
\text { и характеристиках надежности изделий }\end{array}$ \\
\hline
\end{tabular}

Планирование деятельности предприятия, выпускающего сложные наукоемкие изделия в современных условиях связано с необходимостью взаимоувязанных решений по целому ряду направлений, учитывающих возможности:

- перераспределения материальных и людских ресурсов для обеспечения новых заказов, в т.ч. за счет определенной «гибкости» технологических процессов;

- внедрения в процессы разработки, производства, проведения испытаний и эксплуатации изделий современных компьютерных технологий;

- выбора, в рамках действующих финансовых ограничений (лимитной стоимости контракта) поставщиков сырья, материалов, комплектующих изделий, производственного и испытательного оборудования, - в том числе, с учетом постоянного усложнения изделий и прогрессирующего роста их себестоимости;

- развития научно-технического задела под перспективные (еще не финансируемые заказчиком) проекты;

- опережающего выпуска составных частей и комплектующих для производства и ремонта парка изделий;

\section{Baikal Research Journal}


- дальнейшего повышения качества изделия, обеспечения более высоких тактико-технических и эксплуатационно-технических характеристик (в рамках модернизации существующих или разработки новых изделий);

- унификации материалов, комплектующих изделий;

- задействования производственных мощностей, оборудования и стендов для проведения испытаний одновременно для различных образцов изделий;

- перепрофилирования подразделений и специалистов при изменении политики заказов.

Комплексное решение совокупности столь разноплановых задач, в условиях характерного для нынешнего времени динамичного изменения как технологических процессов внутри предприятия (например, за счет появления новых материалов), так и «портфеля заказов», возможно на основе применения и развития методов теории адаптивного управления. Известные трудности практического применения методов адаптивного управления связаны с чрезмерно высокой размерностью исходных данных, необходимых для формализации столь сложных и разноплановых исследуемых процессов, проблемами организации мониторинга множества производственных, технологических и экономических показателей и характеристик деятельности предприятия, рисками гарантированного достижения планируемых показателей проектных разработок и т. д. Таким образом, при очевидной необходимости в управлении процессами ЖЦ сложной наукоемкой продукции внедрение такой системы управления не происходит из-за не разработанности способов формализации и оптимизации всех процессов проектирования сложной наукоемкой продукции (см. рис. 1).

В теории САУ в системах управления входные данные считаются или детерминированными либо изменяющимся по определенным вероятностным законам [4; 5]. В то же время, недостаток информации о текущем состояния всех процессов разработки и производства, а также о воздействиях внешней среды определяют необходимость синтеза системы управления ЖЦ продукции как системы, которая адаптирована по входу, но без ограничений на параметры внешней среды.

В общем виде проблема формирования системы управления процессами жЖЦ сложной наукоемкой продукции сводится к следующему. Возьмем за объект управления совокупность задаваемых параметрами $\left(\varphi_{1}(t), \varphi_{2}(t), \ldots, \varphi_{m}(t)\right)$ связанных между собой технологических, производственных, и экономических процессов разработки проектов, испытаний, выпуска и применения продукции. При этом одни из перечисленных параметров связаны с отдельными стадиями жЦ продукции, другие могут быть связаны с процессами на ряде стадий (рис. 1). Определение совокупности параметров, их взаимных связей, диапазонов их воз значений является отдельной научной проблемой. На параметры объекта $\left(\varphi_{1}(t)\right.$, $\left.\varphi_{2}(t), \ldots, \varphi_{m}(t)\right)$ воздействуют входные $r=r(t)$ и управляющие параметры $u=u(t)$. Обозначим через $\Phi=\Phi\left(\varphi_{1}(t), \varphi_{2}(t), \ldots, \varphi_{m}(t)\right)$ выходные параметры (рис. 3$)$.

Тогда цель адаптивного управления может быть задана следующей системой

$$
\left\{\begin{array}{c}
\Phi\left(\phi_{1}(t), \phi_{2}(t), \ldots, \phi_{m}(t)\right) \geq \alpha ; \\
t_{k+1}-t_{k} \leq t_{\text {зад }},
\end{array}\right.
$$

где а - заданные требования к показателю; $\Phi\left(\varphi_{1}(t), \varphi_{2}(t), \ldots, \varphi_{m}(t)\right)$ - обобщенный функционал состояния технологических, производственных и экономических процессов проектирования, разработки, испытаний и применения продукции; $\left(t_{k+1}-t_{k}\right)-$ промежуток времени, необходимый для перехода из состояния $k$ в состояние $k+1 ; t_{\text {зад }}$ - заданное время переходного состояния.

\section{Baikal Research Journal}

электронный научный журнал Байкальского государственного университета 


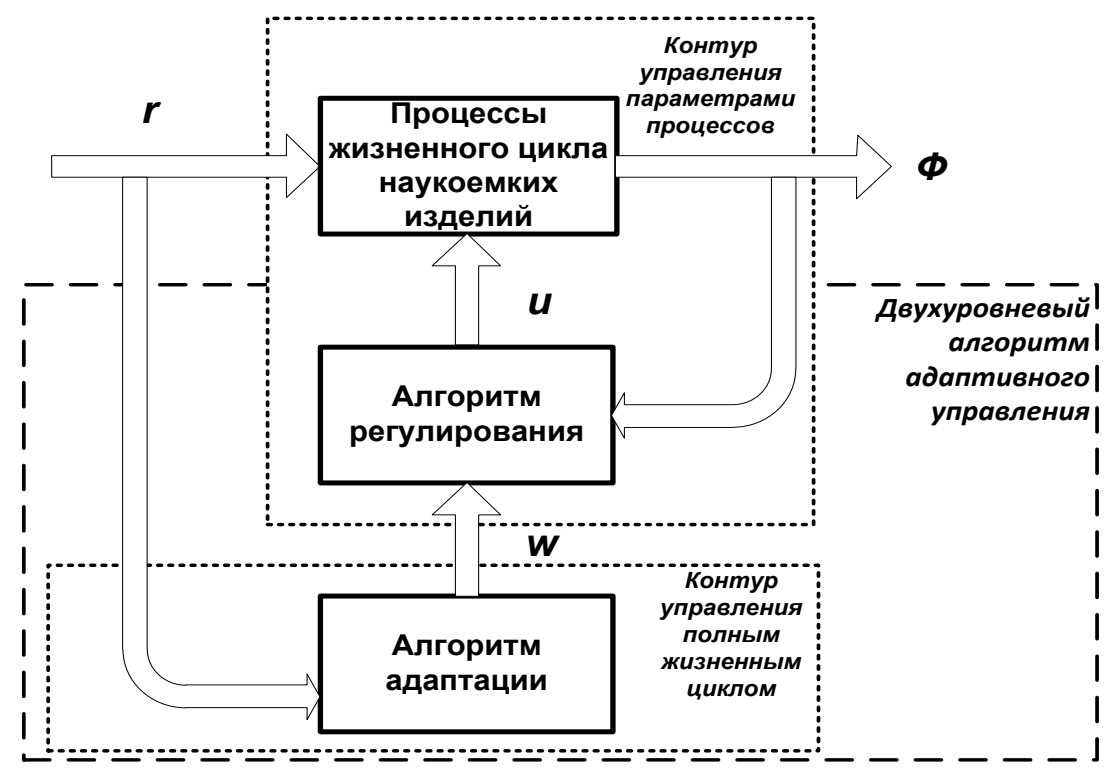

Рис. 3. Структура систелы адаптивного управления процессами жЦ наукоемких изделий

С учетом всех особенностей различных процессов при проектировании, испытаниях, выпуске и применения продукции, рассматриваемая система адаптивного управления представляет собой систему, имеющую двухуровневую структуру (см. рис. 3). Алгоритм регулирования $u$ (алгоритм первого уровня) обеспечивает удовлетворение цели управления, основываясь на настройке параметров объекта в соответствии с управляющими параметрами $w$, т. е. $u=f(\Phi, w)$. Алгоритм адаптации (алгоритм второго уровня) изменяет вектор управляющих параметров $w$ так, чтобы удовлетворить цели управления при заранее неизвестных входных параметрах $r$. Здесь при синтезе алгоритма адаптации используется множество $u^{0}$ (множество установочных параметров).

В теории САУ рассмотренная система с адаптацией по входу в общем виде исследовалась, по всей видимости, только в [5;6]. В современных обзорных работах по теории адаптивных систем, например, в [7; 8] авторы критически относятся $к$ системам с адаптацией по входному сигналу и предлагают, по сути, новую классификацию адаптивных систем, включающую независимые классы систем [7]:

1) с адаптацией по помехе;

2) с применением эталонной модели объекта управления;

3) со стабилизацией параметров основного контура (без идентификации объекта управления);

4) с идентификатором объекта управления (или с уточняемой его моделью);

5) с блоком прогноза выхода объекта управления;

6) с адаптацией, связанной с ошибкой управления;

7) экстремальные системы.

Вместе с тем, в соответствии с такой классификацией, предлагаемая на рис. 3 структура системы адаптивного управления является, по сути, гибридом классов 1 (с адаптацией по помехе) и 4 (с идентификатором объекта, или с уточняемой его моделью). Указанное обстоятельство позволяет сделать общий вывод о том, что существенная трансформация современных объектов (процессов) управления,

\section{Baikal Research Journal}


к которым в полной мере относятся и процессы жизненного цикла сложных наукоемких изделий, стимулирует появление существенно новых структур адаптивных систем.

При вариации входных параметров (например, заказов на проектирование и выпуск новой продукции) или временных показателей планирования деятельности предприятия также может изменяться и цель управления процессами ЖЦ продукции. Это приводит к изменению результатов решения задачи формирования системы управления. Поэтому организация адаптивного управления ЖЦ сложных наукоемких изделий сводится к формированию совокупности алгоритмов управления всех процессов деятельности предприятия. При этом эти алгоритмы должны быть согласованными между собой.

Таким образом, неопределенность заказов на выпуск новых наукоемких изделий, являющихся входными параметрами) ставит более сложную постановку проблемы формирования системы адаптивного управления процессами ЖЦ наукоемкой продукции, отличающуюся отсутствием ограничений на детерминированность и замкнутость множества входных воздействий. Решение такой проблемы даст возможность обеспечить планирование деятельности предприятий в долгосрочной перспективе для более эффективного управления процессами ЖЦ наукоемкой продукции, а также оптимальное применение производственных мощностей и всех ресурсов в среднесрочной и краткосрочной перспективе.

\section{Список использованной литературы}

1. Астахов С. А. Организация адаптивного управления процессами жизненного цикла парашютных систем / С. А. Астахов // Вестник академии военных наук. - 2015. № 3. - C. 151-155.

2. Анализ состояния исследований проблем управления жизненным циклом искусственно созданных объектов / Р. М. Юсупов [и др.] // Труды СПИИРАН. - 2011. № 1 (16). - C. 37-109.

3. Стародубов В. А. Управление жизненным циклом изделий, от концепции до реализации / В. А. Стародубов. - СПб. : Стерлинг Групп, 2006. - 120 с.

4. Репин В. Г. Статистический синтез при априорной неопределенности и адаптация информационных систем / В. Г. Репин, Г. П. Тартаковский. - М. : Сов. радио, 1977. $432 \mathrm{c}$.

5. Фрадков А. Л. Адаптивное управление в сложных системах: беспоисковые методы / А. Л. Фрадков. - М. : Наука, 1990. -296 с.

6. Оценивание качества функционирования автоматизированных систем при параметрической неопределенности внешних воздействий / Воробьев А. А. [и др.] // Безопасность информационных технологий. - 2004. - № 3. - С. 58-62.

7. Жмудь В. А. Адаптивные системы автоматического управления / В. А. Жмудь, Д. О. Терешкин, О. В. Прыткова // Сборник научных трудов НГТУ. - 2011. - № 1 (63). C. $23-40$.

8. Жмудь В. А. Адаптивные системы автоматического управления с единственным основным контуром / В. А. Жмудь // Автоматика и программная инженерия. - 2014. № 2 (8). - C. 106-122.

\section{References}

1. Astakhov S. A. Organization of adaptive control of parachute system life cycle processes. Vestnik akademii voennykh nauk $=$ Bulletin of Academy of Military Sciences, 2015, no. 3, pp. 151-155. (In Russian)

2. Yusupov R. M., Sokolov B. V., Ptushkin A. I., Ikonnikova A. V., Potryasayev S. A., Civirko E. G. State analysis of research on the problems of managing life cycle of artificially created objects. Trudy SPIIRAN = SPIIRAS Proceedings, 2011, no. 1 (16), pp. 37-109. (In Russian).

\section{Baikal Research Journal}


3. Starodubov V. A. Upravlenie zhiznennym tsiklom izdelii, ot kontseptsii do realizatsii [Life cycle management of products, from concept to implementation]. Saint Petersburg, Sterling Grupp Publ., 2006. 120 p.

4. Repin V. G., Tartakovsky G. P. Statisticheskii sintez pri apri-ornoi neopredelennosti $i$ adaptatsiya informatsionnykh system [Statistical synthesis with a priori uncertainty and adaptation of information systems]. Moscow, Sovetskoye radio Publ., 1977. 432 p.

5. Fradkov A. L. Adaptivnoe upravlenie $v$ slozhnykh sistemakh: bespoiskovye metody [Adaptive control in complex systems: searchless methods]. Moscow, Nauka, 1990. 296 p.

6. Vorobyev A. A. Assessing the quality of automated system functioning in terms of parametric uncertainty of external influences. Bezopasnost Informatsionnykh Tekhnologiy = IT Security, 2004, no. 3, pp. 58-62. (In Russian)

7. Zhmud V. A., Tereshkin D. O., Pritkova O. V. Adaptive automatic control systems. Sbornik nauchnykh trudov NGTU = Collection of Scientific Works of Novosibirsk State Technical University, 2011, no. 1 (63), pp. 23-40. (In Russian).

8. Zhmud V. Adaptive automatic control systems with a single primary loop. Avtomatika $i$ programmnaya inzheneriya = Automatics and Software Enginery, 2014, no. 2 (8), pp. 106122. (In Russian).

\section{Информация об авторах}

Воробъев Альберт Анатольевич - д.т.н., с.н.с., ведущий научный сотрудник, лаборатория информационных технологий в системном анализе и моделировании, ФГБУН Санкт-Петербургский институт информатики и автоматизации Российской академии наук (СПИИPAН), 14-я линия, д. 39, Санкт-Петербург, 199178, РФ; e-mail: maestro265@yandex.ru.

Данеев Алексей Васильевич - д.т.н., профессор, профессор кафедры «Информационные системы и защита информации» ФГБОУ ВО «Иркутский государственный университет путей сообщения» (ИрГУПС), Чернышевского ул., 15, Иркутск, 664074, РФ; е-mail: daneev@mail.ru.

\section{Author}

Albert A. Vorobyev - Doctor habil. in Engineering, Leading Researcher, Information Technology Laboratory in System Analysis and Modeling, St. Petersburg Institute of Computer Science and Automation of the Russian Academy of Sciences (SPIIRAS), 39 14th line, 199178, St. Petersburg.

Alexey V. Daneyev - Doctor habil. in Engineering, Professor, Chair of Information Systems and Information Protection, Irkutsk State Railway Engineering University, 15 Chernyshevsky St., 664074, Irkutsk; e-mail: daneev@mail.ru.

\section{Для цитирования}

Воробьев А. А. Организация деятельности предприятия по управлению жизненным циклом сложных наукоемких изделий / А. А. Воробьев, А. В. Данеев // Baikal Research Journal. — 2017. — T. 8, № 4. — DOI : 10.17150/2411-6262.2017.8(4).11.

\section{For Citation}

Vorobyev A. A., Daneyev A. V. Activity arrangement of the enterprise in managing the life cycle of complex high-tech products. Baikal Research Journal, 2017, vol. 8, no. 4. DOI: 10.17150/2411-6262.2017.8(4).11. (In Russian).

\section{Baikal Research Journal}

